\title{
THE EFFECTIVENESS OF REHABILITATION INTERVENTION ON KNEE RECOVERY AMONG PATIENTS WITH LIMITED MOBILITY AFTER LOWER EXTREMITY INJURIES AT NAM DINH GENERAL HOSPITAL IN 2021
}

\author{
Le Xuan Thang', Doan Thi Thu Ha', La Khac Thang', \\ Dong Van Anh'2, Nguyen Thi Hanh ${ }^{2}$ \\ ${ }^{1}$ Nam Dinh University of Nursing; ${ }^{2}$ Nam Dinh General Hospital
}

\begin{abstract}
Objectives: To evaluate the effectiveness of rehabilitation intervention on knee recovery among patients with limited mobility after lower extremity injuries. The results from this study contributed to improving the understanding of the rehabilitation process on patients with limited knee mobility after surgery. Method: a quasi-experimental design study was conducted to compare the differences between before and after rehabilitation intervention from January 2021 to September 2021. 23 patients were treated at the Rehabilitation Department in Nam Dinh General Hospital were selected to participate in the study. Results: $65,2 \%$ of injuries were caused by traffic accidents, $8,7 \%$ were due to work accidents and $8.7 \%$ were due to daily-life accidents. The time from surgery to rehabilitation was: $43,5 \%$ below 1 month, 4,3\% over 6 months. There was a significant change in the limb size difference between the operated leg and the normal leg. After applying rehabilitation intervention, all patients had an improvement in joint range of motion, muscle grade, muscle function according to Lysholm scale, pain level according to VAS scale before and after treatment, especially in cases of functional recovery less than 1 month after surgery. Conclusions: The rehabilitation effectiveness was good after patients were applied rehabilitation methods such as Paraffin, Electropulse, and Motion therapy due to the improvement in range of motion (ROM), muscle level, muscle function according to Lysholm scale, pain level according to VAS scale early after surgery.
\end{abstract}

Keywords: Rehabilitation, knee recovery, limited mobility, lower extremity injury

\section{INTRODUCTION}

Limiting the range of motion of the knee joint after a lower extremity injury causes inconvenience, reduces working capacity, and affects the patient's quality of life. The cause of knee stiffness is due to lower extremity trauma (internal or extra-articular fracture, ligament damage, meniscus) $[1,2,3]$.
Currently, with the strong development of medicine, there are many methods of treating a limited range of motion such as simple rehabilitation, surgery to remove ankylosing knee joint, and arthroscopic surgery to remove ankylosing knee. Rehabilitation is an important part of treatment, and rehabilitation programs are recommended immediately after surgery.
Cor. author: Le Xuan Thang

Address: Nam Dinh University of Nursing

Email: lexuanthangnd@gmail.com
Received: Feb 08, 2022

Accepted: Mar 06, 2022

Published: Mar 07, 2022 
Rehabilitation after surgery plays an important role in helping patients reduce pain, reduce swelling, prevent joint adhesions, facilitate metabolism, and mineral salts to help bones heal quickly and restore mobility, joint movement, increase muscle strength, easy movement, recovery of limb activities after surgery [4].

Restriction of motion of the knee joint after surgery is a common disease with many degrees of manifestation in one of three forms: limitation of flexion, limitation of extension, or limitation of both flexion and extension. Regardless of the type and extent, this sequelae is a nuisance in daily life, reduces working capacity, and affects the patient's quality of life.

Whether the function of the injured limb is fully restored or not depends in part on the correct care and exercise after surgery. The secondary injuries caused by immobilization are usually knee stiffness, limited range of motion, muscle atrophy, and edema, the rate of knee mobility restriction account for $40 \%$, and muscle atrophy and edema accounted for $20 \%$ [5]. Therefore, the rehabilitation of the knee joint for patients after surgery plays an important role, so it should be done as soon as possible. Knee rehabilitation techniques help reduce the risk of stiffness, increase muscle strength and help patients with rehabilitation early to move and move easily. To contribute to improving the understanding of the rehabilitation process in patients with limited knee mobility after surgery, we carried out the topic: "the effectiveness of rehabilitation intervention on knee recovery among patients with limited mobility after lower extremity injuries at Nam Dinh general hospital in 2021" with the goal: To evaluate the effectiveness of rehabilitation intervention on knee recovery among patients with limited mobility after lower extremity injuries.

\section{RESEARCH SUBJECTS AND METHODS}

\subsection{Research subjects}

All patients diagnosed with limited knee mobility after lower extremity injuries were treated at the Department of Rehabilitation of Nam Dinh General Hospital in 2020.

\subsubsection{Selection criteria}

- Patients with limited movement of the knee joint, limited flexion and extension of the knee joint, or both, accompanied by knee pain affecting daily activities.

- Had a history of trauma to the thigh, knee, lower leg.

- Had surgical treatment.

- The patient was diagnosed with limited knee mobility after a lower extremity injury, the patient needs to improve the range of flexion-extension of the knee joint.

- Have a complete and clear medical record.

\subsubsection{Exclusion criteria}

- Patient was not consciousness, was not able to make active movements.

- Patients with other injuries combined: damage to blood vessels, nerves.

- Pathological fractures, limited knee movement after knee replacement, knee arthritis, knee osteoarthritis.

- Patients did not complete the rehabilitation intervention.

\subsection{Duration and setting}

- Research period: from January 2021 to September 2021. 
- Setting: Department of Rehabilitation Nam Dinh General Hospital.

\subsection{Research design}

- A quasi-experimental design study was conducted to compare the differences before and after rehabilitation intervention.

- Sample size and sampling: The study sample was selected by convenient sampling method, in turn selecting patients diagnosed with limited knee mobility after lower extremity injuries treated at the Department of Rehabilitation - Nam Dinh General Hospital, at that time period from January 2021 to December 2021.

23 patients diagnosed with limited knee mobility after surgery met the inclusion and exclusion criteria.

Clinical examination, $\mathrm{X}$-ray of the knee joint, ultrasound of the knee joint.

\section{Research targets:}

1. The difference in limb size between the operated leg and the healthy leg

2. Pain according to the VAS scale

3. Measure the range of motion of the knee joint according to the Zero method

4. Measurement of mechanical force

5. Evaluation of knee function according to Lysholm scale (Lysholm Knee Scale 1985)

\section{Patients had prescribed rehabilitation methods:}

1. Bitter paraffin in knee joint 20 minutes/ time/day

2. Electrical pulse size muscle in thighs, shins 15 minutes/time/day

3. Exercise with knee support once a day
Data analysis, comparison at the time points before treatment (D0), after 15 days of treatment (D15) of treatment. Evaluation of treatment results

\subsection{Research media}

- The form to collect information about the patient with limited knee mobility after orthopedic injury (Form M1) is used to interview the patient and the patient fills in the information.

- Rehabilitation evaluation form is evaluated and filled in by doctors and Rehabilitation therapists.

- Scale to assess pain level.

- Evaluation table according to Lysholm scale.

- Measuring range of motion joint MN-ROM (Ranger of Motion) of Phana company, tape measure.

- Electrolysis, Physiotherapy \& Rehabilitation ES-520

- Paraffin cooking pot

\subsection{Ethics in research}

- The research was approved by the Ethics Committee in Nam Dinh University of Nursing and permission from the Manager Board of the Department of Rehabilitation.

- Patients were clearly explained about the research purpose and voluntarily participate in the research program. Patient information was kept confidential and was only used for research purposes.

- During the course of treatment, if the patient did not get better or gets worse, the patient may be stopped from the study, changed the treatment method, and removed from the study group. 


\section{RESULTS}

\subsection{General features}

\subsubsection{Time from after surgery to the time the patient was rehabilitated}

Patients who received early rehabilitation training for less than 1 month accounted for the highest rate of $43.5 \%$, the lowest rate of $4.3 \%$ over 6 months.

\subsubsection{Rehabilitation method specified}

Table 1. Rehabilitation method specified

\begin{tabular}{|lcc|}
\hline \multicolumn{1}{|c}{ Rehabilitation method } & Amount (n) & Ratio (\%) \\
\hline Heat Therapy (Paraffin) & 23 & 100 \\
\hline Electrotherapy (Electrical Muscle Stimulation) & 20 & 87 \\
\hline Motion therapy & 23 & 100 \\
\hline
\end{tabular}

Interpretation: In the study, 23 patients were assigned heat therapy and exercise, 20 were assigned electrotherapy. There were 3 people who were not assigned to electrotherapy because the patient still had a bone fusion device.

\subsection{Clinical characteristics of study subjects before and after treatment}

3.2.1. Change in the limb size difference between surgical leg and healthy leg before and after treatment

Table 2. Evaluation of the difference in the limb size difference between the surgical leg and the healthy leg

\begin{tabular}{|c|c|c|c|c|c|}
\hline \multirow{3}{*}{ Body size difference } & \multicolumn{4}{|c|}{ Research group } & \multirow{3}{*}{$p_{1-2}$} \\
\hline & \multicolumn{2}{|c|}{ Before treatment (1) } & \multicolumn{2}{|c|}{ After treatment (2) } & \\
\hline & $\mathbf{n}$ & $\%$ & $\mathbf{n}$ & $\%$ & \\
\hline No change: $<=2 \mathrm{~cm}$ & 8 & 34,8 & 10 & 43,5 & \multirow{3}{*}{$p=0,426>0,05$} \\
\hline $\begin{array}{l}\text { Changes (muscle atrophy, } \\
\text { edema) }>2 \mathrm{~cm}\end{array}$ & 15 & 65,2 & 13 & 56,5 & \\
\hline Mea $n \pm S D$ & \multicolumn{2}{|c|}{$1,35 \pm 0,487$} & \multicolumn{2}{|c|}{$1,43+0,507$} & \\
\hline
\end{tabular}

Interpretation: The mean results of the 2 variables of limb size difference before and after treatment are 1.35 and 1.43 , respectively, and the standard deviation is 0.487 and 0.507 (The difference was not statistically significant $(>0.05)$. 


\subsubsection{Change in knee range of motion before and after treatment}

Table 3. Evaluation of the change of range of motion in flexion and extension of the knee joint

\begin{tabular}{|c|c|c|c|c|c|}
\hline \multirow{3}{*}{ Motion } & \multirow{3}{*}{$\begin{array}{c}\text { Knee joint range } \\
\text { of motion }\end{array}$} & \multicolumn{4}{|c|}{ Research group } \\
\hline & & \multicolumn{2}{|c|}{ Before treatment (1) } & \multicolumn{2}{|c|}{ After treatment (2) } \\
\hline & & $\mathbf{n}$ & $\%$ & $\mathbf{n}$ & $\%$ \\
\hline \multirow{4}{*}{ 1. Knee flexic } & $\geq 120^{\circ}$ & 0 & 0 & 4 & 17,4 \\
\hline & {$\left[90^{\circ}, 120^{\circ}\right)$} & 4 & 17,4 & 13 & 56,5 \\
\hline & {$\left[60^{\circ}, 90^{\circ}\right)$} & 13 & 56,5 & 4 & 17,4 \\
\hline & $<60^{\circ}$ & 6 & 26,1 & 2 & 8,7 \\
\hline \multicolumn{2}{|c|}{ Total } & 23 & 100 & 23 & 100 \\
\hline \multicolumn{2}{|c|}{ Mean \pm SD } & \multicolumn{2}{|c|}{$3,09 \pm 0,668$} & \multicolumn{2}{|c|}{$2,17 \pm 8,34$} \\
\hline \multicolumn{2}{|c|}{$p_{1-2}$} & \multicolumn{4}{|c|}{$p=0,000<0,05$} \\
\hline \multirow{5}{*}{ 2. Knee extenti } & {$\left[0^{0}, 5^{0}\right]$} & 17 & 73,9 & 19 & 82,6 \\
\hline & {$\left[-5^{0}, 0^{0}\right]$} & 6 & 26,1 & 4 & 17,4 \\
\hline & & & & & \\
\hline & {$\left[-10^{0},-5^{0}\right]$} & 0 & 0 & 0 & 0 \\
\hline & $<-10^{0}$ & 0 & 0 & 0 & 0 \\
\hline \multicolumn{2}{|c|}{ Total } & 23 & 100 & 23 & 100 \\
\hline \multicolumn{2}{|c|}{ Mean \pm SD } & \multicolumn{2}{|c|}{$1.26 \pm 0,449$} & \multicolumn{2}{|c|}{$1,17 \pm 0,388$} \\
\hline \multicolumn{2}{|c|}{$\mathbf{p}_{1-2}$} & \multicolumn{4}{|c|}{$p=0,328>0,05$} \\
\hline
\end{tabular}

Interpretation: - After 15 days of treatment, the range of motion of knee flexion has improved markedly, the average range of motion from $3.09 \pm 0,668$ reduced to $2,17 \pm 8,34$, the difference was not statistically significant $(p<0,05)$.

-Knee extensor range of motion improved, the mean value from $1.26 \pm 0.449$ decreased to $1,17 \pm 0,388$, the difference between the two variables was not statistically significant $(p>0,05)$. 


\subsubsection{Changes in muscle level before and after treatment}

Table 4. Evaluation of the change in the fundamental order

\begin{tabular}{|c|c|c|c|c|c|}
\hline \multicolumn{6}{|c|}{ Research group } \\
\hline \multirow[t]{2}{*}{ Muscle level } & \multicolumn{2}{|c|}{ Before treatment (1) } & \multicolumn{2}{|c|}{ After treatment(2) } & \multirow[t]{2}{*}{$\mathbf{p}_{1-2}$} \\
\hline & $\mathbf{n}$ & $\%$ & $\mathbf{n}$ & $\%$ & \\
\hline Level 0 & 0 & 0 & 0 & 0 & \multirow{8}{*}{$p=0,000<0,05$} \\
\hline Level 1 & 0 & 0 & 0 & 0 & \\
\hline Level 2 & 1 & 4,3 & 0 & 0 & \\
\hline Level 3 & 8 & 34,8 & 0 & 0 & \\
\hline Level 4 & 13 & 56,5 & 10 & 43,5 & \\
\hline Level 5 & 1 & 4,3 & 13 & 56,5 & \\
\hline Total & 23 & 100 & 23 & 100 & \\
\hline Mean \pm SD & \multicolumn{2}{|c|}{$3,61 \pm 0,656$} & \multicolumn{2}{|c|}{$4,57 \pm 0,507$} & \\
\hline
\end{tabular}

Interpretation: The muscle class of the study group before and after treatment had a marked improvement, the mean value increased from 3.61 up to $4,57 \pm 0,507$, the difference was not statistically significant $(p<0,05)$.

\subsubsection{Change of knee joint function according to Lysholm scale}

Table 5. Assessment of changes in knee function according to Lysholm scale

\begin{tabular}{|c|c|c|c|c|c|}
\hline \multirow{3}{*}{$\begin{array}{l}\text { Knee function } \\
\text { according to } \\
\text { Lysholm scale }\end{array}$} & \multicolumn{4}{|c|}{ Research group } & \multirow{3}{*}{$\mathbf{p}_{1-2}$} \\
\hline & \multicolumn{2}{|c|}{ Before treatment (1) } & \multicolumn{2}{|c|}{ After treatment(2) } & \\
\hline & $\mathbf{n}$ & $\%$ & $\mathbf{n}$ & $\%$ & \\
\hline Poor (<65 points) & 17 & 73,9 & 4 & 17,4 & \multirow{6}{*}{$p=0,000<0,05$} \\
\hline Average (65-83 points) & 6 & 26,1 & 16 & 69,6 & \\
\hline Rather (84-94 points) & 0 & 0 & 1 & 4,3 & \\
\hline Good (95-100 points) & 0 & 0 & 2 & 8,7 & \\
\hline Total & 23 & 100 & 23 & 100 & \\
\hline Mean \pm SD & \multicolumn{2}{|c|}{$3,74 \pm 0,449$} & \multicolumn{2}{|c|}{$2,96 \pm 0,767$} & \\
\hline
\end{tabular}

Interpretation: Knee function according to Lysholm scale before and after treatment had a clear improvement, the mean value from $3.74 \pm 0.449$ decreased to $2.96 \pm 0.767$, the difference between the two variables was statistically significant $(p) .<0.05)$. 


\subsubsection{VAS scale of pain}

Table 6. Evaluation of the change of pain level according to the VAS scale before and after treatment

\section{Research group}

\begin{tabular}{|c|c|c|c|c|c|}
\hline \multirow[t]{2}{*}{ Pain level VAS } & \multicolumn{2}{|c|}{ Before treatment (1) } & \multicolumn{2}{|c|}{ After treatment(2) } & \multirow[t]{2}{*}{$\mathbf{p}_{1-2}$} \\
\hline & $\mathbf{n}$ & $\%$ & $\mathbf{n}$ & $\%$ & \\
\hline No pain (0 point) & 1 & 4,3 & 5 & 21,7 & \multirow{5}{*}{$p=0,000<0,05$} \\
\hline Little pain (1-3 points) & 7 & 30,4 & 15 & 65,2 & \\
\hline $\begin{array}{l}\text { Moderate pain } \\
\text { (4-6 points) }\end{array}$ & 14 & 60,9 & 3 & 13 & \\
\hline $\begin{array}{l}\text { A lot of pain } \\
\text { (7-10 points) }\end{array}$ & 1 & 4,3 & 0 & 0 & \\
\hline Mean \pm SD & \multicolumn{2}{|c|}{$2,65 \pm 0,647$} & \multicolumn{2}{|c|}{$1,91 \pm 0,596$} & \\
\hline
\end{tabular}

Interpretation: The level of pain according to the VAS scale before and after treatment had a marked improvement, the mean value from $2.65 \pm 0.647$ decreased to $1.91 \pm 0.596$, the difference between the two variables was statistically significant $(p<0.05)$.

\section{DISCUSSION}

After the injury, the patient was operated on early. After surgery, early rehabilitation exercise is essential to limit complications (pain, edema) and sequelae of limited knee joint mobility, reducing mobility, mobility as well as difficulty in walking the patient's daily activities.

Patients undergoing rehabilitation treatment until late (1-3 months) of the knee joint often have limited movement of flexion and extension, difficulty in moving and daily activities. To support favorable exercise, physical therapy methods also play a very important role: Paraffin technique helps to relieve pain, relax muscles, dilate peripheral vessels, increase local circulation; Electroneuromuscular stimulation technique. In the department, besides exercise, patients are assigned these two techniques and bring good treatment results.

Patients with limited knee movement after 15 days of treatment at the Rehabilitation Department Knee joints have markedly improved, namely:

Improvement in joint range of motion: Before treatment, there was no case of knee joint range of motion $\geq 1200,17.4 \%$ of cases had a range of motion from [900, 1200 ], $56.5 \%$ of cases with ROM from [600, 900 ], $26.1 \%$ of cases with ROM $<600$. After treatment, $17.4 \%$ of cases with ROM of the knee joint $\geq 1200,56.5 \%$ of cases with ROM of the knee joint from $[900,1200]$.

The average knee flexion range of motion decreased from $3.09 \pm 0.668$ to $2.17 \pm 8.34$. This result was higher than the study of Nguyen Hoang Long (2006) on 30 patients after surgery in the immobilized 
stage, $16.7 \%$ of patients had a joint range of motion $\geq 1200,43.3 \%$ achieved from $900,1200], 13.3 \%$ achieved from [600, 900 ], and $26.7 \%$ of patients had a joint range of motion < 600 [5 6] and lower than Nguyen The Thi 2002) in the period after immobilization 4 weeks after surgery) knee flexion range of motion reached 1200 with $77.4 \%$, from $[900,1200]$ accounted for $12.9 \%$, from $[600,900]$ accounted for $9.7 \%$ and no patients with knee range of motion less than $600[2,6]$.

Improvement in muscle grade: Before treatment, $4.3 \%$ of patients had second grade, $34.8 \%$ of third, $56.5 \%$ of fourth and $4.3 \%$ of fifth. After treatment $43.5 \%$ of patients with grade 4 muscle disease and $56.55 \%$ with grade 5 muscle. The mean muscle grade increased from $3.61 \pm 0.656$ to $4.57 \pm 0.507$.

This result is lower than the study of Huynh Thanh Long et al. A cross-sectional descriptive study on 30 patients with limited knee mobility after injury showed an improvement in thigh muscle strength compared to before. $30 \%$ of patients have good muscle strength after treatment, $66.7 \%$ have good muscle strength after treatment. Classification according to the Lysholm and Gillquist scale has markedly improved after treatment[1].

Improvement in knee function according to Lysholm scale: Before treatment, $73.9 \%$ of patients had poor knee function according to Lysholm scale, and $26.1 \%$ achieved average. After treatment, $17.4 \%$ of patients had poor knee function according to Lysholm scale, $69.6 \%$ got average, $4.3 \%$ got good, $8.7 \%$ got good; Knee function according to Lysholm scale has the average value from $3.74 \pm 0.449$ to 2.96 \pm 0.767 . Thus, knee function has improved significantly compared to before treatment, the difference is statistically significant due to $p<0.05$.

The improvement of pain level according to the VAS scale: Before treatment, $4.3 \%$ of patients had no pain, $30.4 \%$ had little pain, $60.9 \%$ had moderate pain, $4.3 \%$ had a lot of pain. After treatment, $21.7 \%$ of patients had no pain, $65.2 \%$ of patients had little pain, $13 \%$ had moderate pain, and there was no case of severe pain. The pain level according to the VAS scale has an average value from $2.65 \pm 0.647$ to $1.91 \pm 0.596$. The study results showed that the pain level decreased gradually over time, the difference was statistically significant due to $p<0.05$.

Thus, after 15 days of rehabilitation, the knee joint had a clear improvement, which showed the importance of using rehabilitation methods in the treatment of patients after knee surgery in general and people with disabilities with posttraumatic illness in particular. Patients need to be guided and consulted early on rehabilitation treatment methods. Patients practice and use physical therapy methods to help reduce pain, increase joint range of motion, improve muscle strength in the lower extremities and soon reintegrate into society.

\section{CONCLUSION}

By monitoring 23 patients diagnosed with limited knee mobility after lower extremity injuries treated at the rehabilitation department from January 2020 to September 2020, it was found that: - Patients receiving early rehabilitation treatment less than 1 month accounted for 
the highest rate of $43.5 \%$, the lowest rate of $4.3 \%$ over 6 months.

- 23 patients had applied the method of motor rehabilitation therapy (exercise), therapy (electric impulse muscle stimulation), heat therapy (paraffin application).

- Good rehabilitation effect due to the improvement in joint range of motion, muscle level, muscle function according to Lysholm scale, pain level according to VAS scale before and after treatment, the difference was statistically significant with $p$ $<0.05$.

After treatment: $17.4 \%$ of cases of knee osteoarthritis $\geq 1200,56.5 \%$ of cases of knee osteoarthritis from [900,1200], 17.4\% of cases with osteoarthritis from [600,900], $8,7 \%$ of cases have TVD $<600 ; 43.5 \%$ of patients with grade 4 muscle disease and 56.55 grade 5 muscle disease; $17.4 \%$ of patients with knee function according to Lysholm scale achieved poor grade, $69.6 \%$ achieved average grade, $4.3 \%$ achieved good grade, $8.7 \%$ achieved good grade $; 21.7 \%$ of patients had no pain, $65.2 \%$ of patients had little pain, $13 \%$ had moderate pain and there was no case of severe pain.

This result showed that rehabilitation plays an important role in maintaining and improving motor function and independent mobility of patients with limited knee mobility after lower extremity injury.

\section{REFERENCES}

1. Dauty M, et al (2007). "Physical training in rehabilitation programs before and after total hip and knee arthroplasty". Ann Readapt Med Phys";50:
462-8, 455-61. https://doi.org/10.1016/j. annrmp.2007.04.011

2. Kevin E. Wilk, et al (2006), "Rehab of Articular Leison in the Athlete"s Knee", Article journal of Orthopaedic and Sports Physical Therapy, November 2006. https:// www.jospt.org/doi/10.2519/jospt.2006.2303

3. Nguyen The Thi (2002), "Evaluation of wounds, treatment of early knee soft wounds at Viet Duc hospital", Master of Medicine Thesis, Hanoi Medical University, pp. 62-64.

4. Nguyen Hoang Long (2006, Developing and evaluating the effectiveness of preventing knee stiffness complications of the guideline for movement therapy for patients after fusion surgery due to closed $1 /$ lower thigh fracture by method braces, Bachelor of Medicine Thesis, Hanoi Medical University, pp. 38-45.

5. Ediz L, et al (2012), "A randomized controlled trial of electrostimulation effects on effusion, swelling and pain recovery after anterior cruciate ligament reconstruction": a pilot study", Clin Rehabil.; 26:413-42. https://doi.org/10.1177/0269215511421029

6. Huynh Thanh Long et al (2015): "Evaluating the results of treatment of limited knee mobility after lower extremity injury by stretching method at the Can Tho Orthopedic And Rehabilitation Center in 2014-2015 “. 